\title{
Quand faut-il opérer les diverticulites?
}

Entre la diverticulite sigraoïdienne à peu près silencieuse, constata-tion radiologique banale, et la péritonite par perforation diverticu-laire au pronostic hautement severe ( 25 morts sur 111 opérés!) toutes les variétés se rencontrent, qui provoquent d'éternelles discussions thérapeutiques entre les consultations de médecine où $\Gamma$ affection pa-raît bien innocente, et les cliniques chirurgicales où elle est considérée comme des plus graves. Un rapport de E. Henry (de Marseille) et G. Edelmann (de Paris) a fait le point de la question au $54^{\circ}$ Congrès de ГAssociation françaíse de chírurgíe (Paris, septembre 1962); nous laissons de côté les descriptions techniques pour retenir ici les indications thérapeutiques qui sont remarquablement nuancées.

La diverticulite simple guérit le plus souvent par le traitement medical, grace aux antibiotiques et aux sulfamidés non résorbables; et le contrôle diététique et médicamenteux suffit à maintenir la guérison dans bon nombre de cas. Mais le tiers des diverticulites se compliquent: faut-il done proposer la côlectomie prophylactíque, operation bénigne, pour éviter d'avoir à imposer une intervention dangereuse en cas de complication? Les rapporteurs s'insurgent avec raison contre cette prétention abusive, et réservent l'opération radicale, à froid, aux formes dont revolution future peut faire craindre la survenue d'une telle complication: crises répétées réagissant mal aux medicaments ou s'ac-compagnant d'hémorragies; persistance d'une masse palpable ou radiologique; etc.

Les diverticulites compliquées, formes essentiellement chirurgicales, reconnaissent des indications thérapeutiques assez variées. Les síg-moïdítes aíguës ( $27 \%$ des cas) exigent une intervention soit immediate - si possible côlectomie immediate, sinon colostomie de dé-charge soit retardée («resection idéale»); les tumeurs ínflammatoíres chroníques $(24 \%)$ seront réséquées en un temps ou après derivation; Гocclusíon par sígmoïdíte est généralement reversible par traitement medical et aspiration, en attendant la resection (pour suspicion de cancer); les hémorragíes massives ou récidivantes sont rares (4,5\% des cas), mais proviennent souvent de lesions étendues à tout le colon; enfin Vassociation de diverticule sígmoïdien et de cancer est un risque trop peu frequent $(6,5 \%)$ pour justifier la resection systématique de toutes les diverticulites; sa mortalité opératoire est élevée (3 sur 22).

\section{Editorial}

\section{7}

Le diagnostic dífférentíel avec le cancer pousse fréquemment à la laparotomie; sous les yeux ou les mains du cbirurgien, la nature de la lesion reste parfois delicate à determiner. Henry et Edelmann recom-mandent alors de pratiquer une colostomie de la region suspecte: comme la dívertículite ne s, accompagne jamais d'une atteinte de la mu-queuse côlíque, Гintégrité de celleci garantit $\Gamma$ absence de dégénéres-cence.

La côlectomíe est la seule méthode qui assure le traitement radical des díverticulítes complíquêes. La colostomie, même conservée de nombreux mois, ne met pas à Гabri des récidives; elle n'est jamais qu'une operation palliative, qui doit être suivie ultérieurement d'une resection. Celle-ci entraîne une mortalité globale de 5 \%. II suffit d"“enlever le sígmoïde; 
Textension des lesions sur le colon descendant n'exige pas l'ablation de celui-ci, car les diverticules ne récidivent pas à ce niveau; d'autre part les diverticules ne descendent jamais sur le rectum, de sorte qu'on peut épargner ce segment. II suffit done d'enlever le sig-moïde, mais ílfaut $\Gamma$ enlever en entier, sous peine de rechute.

La discussion reste ouverte entre les chirurgiens, qui voudraient enlever à froid, avec un minimum de risques, les sigmoïdes qui ont fait $\Gamma$ objet d'une poussée de diverticufite, et les internistes pour lesquels Гaffection est bénigne 9 fois sur 10 au moins. Pour résoudre ce problème, il faudrait reprendre les observations des diverticulites diagnostiquées il y a 10 ans et plus, et s'enquérir sur leur evolution; c'est une etude qui, à notre connaissance n'a jamais été faite, et qui seule apporterait une solution valable à cette question. M. D. 